\title{
DYRK2 is a ciliary kinase involved in vertebrate Hedgehog signal transduction
}

2

Nicholas Morante ${ }^{1}$, Monika Abedin Sigg ${ }^{1}$, Luke Strauskulage ${ }^{1}$, David R. Raleigh ${ }^{2}$ and Jeremy F. Reiter $^{1,3^{*}}$

Affiliations

${ }^{1}$ Department of Biochemistry and Biophysics, Cardiovascular Research Institute, University of California, San Francisco, CA 94158, USA

${ }^{2}$ Department of Radiation Oncology, Department of Neurological Surgery, University of California San Francisco, San Francisco, CA 94158, USA

${ }^{3}$ Chan Zuckerberg Biohub, San Francisco, CA 94158, USA

${ }^{*}$ Correspondence: Jeremy.Reiter@ucsf.edu

\section{ABSTRACT}

Primary cilia are organelles specialized for signaling. We previously defined the proteomes of sea urchin and sea anemone cilia to identify ciliary proteins that predate the origin of bilateria. This evolutionary perspective on cilia identified DYRK2, a kinase not been previously implicated in ciliary biology. We found that DYRK2 localizes to cilia and that loss of DYRK2 disrupts ciliary morphology. We also found that DYRK2 participates in ciliary $\mathrm{Hh}$ signal transduction, communicating between SMO and GLI transcription factors. Mutation of mouse Dyrk2 resulted in skeletal defects reminiscent of those caused by loss of Indian hedgehog (Ihh). Like Dyrk2 mutations, pharmacological inhibition of DYRK2 dysregulates ciliary length control and attenuates Hedgehog signaling. Thus, DYRK2 is required for ciliary morphology, for Hedgehog signaling in vitro, and for skeletal development. We propose that DYRK2 is part of the mechanism that transduces SMO to activate GLI transcription factors within cilia.

\section{INTRODUCTION}

Primary cilia participate in some forms of intercellular communication. One critical signaling pathway transduced via primary cilia is the Hedgehog $(\mathrm{HH})$ pathway. In the absence of $\mathrm{HH}$ ligand, its receptor, Patched1 (PTCH1), localizes to the cilium where it represses $\mathrm{HH}$ activation by blocking accumulation of SMO at the cilium (Corbit, Aanstad et al. 2005, Rohatgi, Milenkovic et al. 2007). Simulating cells with HH or Smoothened Agonist (SAG), a pharmacological SMO agonist (Chen, Taipale et al. 2002), stimulates SMO accumulation at the cilium. Ciliary SMO activates GLI transcription factors, but the molecular mechanisms by which it does so remains unclear.

The $\mathrm{HH}$ signaling pathway has diverse roles in development, including patterning of the neural tube and digits via Sonic hedgehog (SHH) (Hill 1995, Ericson, Briscoe et al. 1997, 
Sharpe, Lettice et al. 1999) and differentiation and proliferation of chondrocytes via IHH (StJacques, Hammerschmidt et al. 1999).

The association of the $\mathrm{HH}$ pathway with primary cilia may be more ancient than the origin of chordates as sea urchins require cilia for $\mathrm{HH}$ signal transduction (Warner, McCarthy et al. 2014). Sea anemones also possess $\mathrm{HH}$ signaling pathway components, although it remains unclear whether they require cilia for $\mathrm{HH}$ signal transduction (Matus, Magie et al. 2008). Correlating with the absence of evidence of DYRK2 in their cilia, choanoflagellates do not possess a canonical HH pathway.

To identify ciliary signal transduction components, we previously defined the choanoflagellate, sea anemone and sea urchin ciliary proteomes and identified signaling regulators not previously associated with cilia (Sigg, Menchen et al. 2017). We detected Dual specificity tyrosine-phosphorylation-regulated kinase 2 (DYRK2) in both the sea anemone and sea urchin ciliary proteomes, but not the choanoflagellate ciliary proteome. DYRK2 is a member of a family of kinases conserved from basal eukaryotes to humans and implicated in diverse processes such as E3 ubiquitin ligase complex formation and Nuclear Factor of Activated Tcells (NFAT) sequestration (Maddika and Chen 2009, Aranda, Laguna et al. 2011).

Inspired by its putative localization in cilia and its strong evolutionary conservation, we sought to elucidate the roles of mammalian DYRK2. Here, we show that DYRK2 is required for ciliary morphogenesis, $\mathrm{HH}$ signaling, and endochondral ossification, revealing DYRK2 to be an essential regulator of ciliary $\mathrm{HH}$ signaling.

\section{RESULTS AND DISCUSSION}

\section{DYRK2 is an evolutionarily conserved ciliary kinase}

Homologs of DYRK2 are present in diverse eukaryotes, and orthologs of mammalian DYRK2 are present in animals, from Trichoplax to humans (Supplementary figure $1 \mathrm{~A}$ and $\mathrm{B}$ ). We recently identified DYRK2 as a member of the ciliary proteomes of sea anemones and sea urchins (Figure 1A) (Sigg, Menchen et al. 2017). The detection of DYRK2 protein in the ciliary proteomes of clades evolutionary distant from mammals prompted us to examine its localization in mammalian cells.

To confirm the proteomics indication that DYRK2 can localize to cilia, we expressed a GFP-tagged form of sea urchin DYRK2 in human retinal pigment epithelium (RPE-1) cells and

67 found that it was enriched in primary cilia (Figure 1B). To determine if mammalian DYRK2 also 68 localizes to cilia, we expressed FLAG-tagged mouse DYRK2 in inner medullary collecting duct 69 (IMCD3) cells and found that it similarly localized to primary cilia (Figure 1C). As kinase dead 
forms of FLAG-tagged DYRK2 also localized to cilia, the serine/threonine kinase activity of mouse DYRK2 is not required for ciliary localization (Figure 1C).

\section{DYRK2 controls ciliary length}

To investigate whether DYRK2 functions in ciliogenesis or ciliary signaling, we used CRISPR-mediated genome editing to mutate Dyrk2 in mouse NIH/3T3 cells. We created two $\mathrm{NIH} / 3 \mathrm{~T} 3$ cell lines (referred to as Mut1 and Mut2), each possessing two deletion alleles of Dyrk2 that alter the reading frame (Supplemental figure 2A). As assessed by immunoblotting for DYRK2, neither Mut1 and Mut2 cell produced detectable levels of DYRK2 (Figure 1D).

To test if DYRK2 is required for ciliogenesis, Mut1, Mut2 and parental wild-type NIH/3T3 cells were grown to confluency and serum starved to induce ciliogenesis. The cells were stained with antibodies against ciliary membrane component ARL13B (Caspary, Larkins et al. 2007) and ciliary axoneme component acetylated tubulin. Dyrk2 mutant cells possessed cilia, indicating that DYRK2 is dispensable for ciliogenesis (Figure 1E). Cilia in both Dyrk2 mutant lines were markedly longer compared to those of the wild-type control cells (Figure 1F). Quantification of cilia length confirmed a mean difference of $1.38 \pm 0.237 \mu \mathrm{m}(\mathrm{p}<0.0001)$ and $2.04 \pm 0.298 \mu \mathrm{m}(\mathrm{p}<0.0001)$ between wild-type cilia and mutant lines Mut1 and Mut2, respectively, indicating that DYRK2 restricts ciliary length.

\section{DYRK2 is required for $\mathrm{HH}$ signaling}

Components of the $\mathrm{HH}$ signal transduction pathway are dispensable for ciliogenesis. As DYRK2 was not required for ciliogenesis, we tested whether it participated in $\mathrm{HH}$ signaling. Loss of DYRK2 in NIH/3T3 cells blocked induction of the HH pathway target genes Gli1 and Ptch1 by SAG suggesting that, contrary to a previous report (Varjosalo, Björklund et al. 2008), DYRK2 positively regulates the $\mathrm{HH}$ pathway (Figure $2 \mathrm{~A}$ and $\mathrm{B}$ ).

Because DYRK2 is required for activation of the $\mathrm{HH}$ pathway by SAG, a direct SMO agonist, DYRK2 acts either at the level of SMO or downstream of it. To determine if DYRK2 regulates accumulation of SMO at the cilium, we analyzed the localization of endogenous SMO in wild type Dyrk2 mutant NIH/3T3 cells. As in wild type NIH/3T3 cells, SMO was absent from cilia in unstimulated Dyrk2 mutant cells and became enriched in cilia upon stimulation with SAG, suggesting that DYRK2 is required for $\mathrm{HH}$ signal transduction downstream of the accumulation of SMO within cilia (Figure $2 \mathrm{C}$ ). 
104

105

106

107

108

109

110

111

112

113

114

115

116

117

118

119

120

121

122

123

124

125

126

127

128

129

130

131

132

133

134

135

136

137

2010). As in wild type NIH/3T3 cells, GLI2 and GLI3 localized to the tips of cilia and became enriched upon stimulation with SAG in Dyrk2 mutant cells (Figure 2D and E)

GLI2 and GLI3 can act as either transcriptional activators or repressors, whereas GLI1 is a dedicated activator (Dai, Akimaru et al. 1999, Sasaki, Nishizaki et al. 1999). To determine if DYRK2 regulates GLI protein levels, we immunoblotted lysates from wild type and Dyrk2 mutant cells that were either unstimulated or stimulated with SAG for GLI1, GLI2 and GLI3. In wild type cells, activation of the $\mathrm{HH}$ pathway increased levels of GLI2, whereas in the absence of DYRK2, pathway stimulation had no effect on GLI2 levels (Figure 2F). Similarly, in the absence of DYRK2, pathway activation had little effect on GLI1 levels (Figure 2F).

In the absence of $\mathrm{HH}$ stimulation, GLI3 is proteolytically processed into a truncated repressor form, and the presence of $\mathrm{HH}$ suppresses this processing (Wang, Fallon et al. 2000). To determine if DYRK2 regulates GLI3 processing, we examined how pathway stimulation affected the levels of full length GLI3 and truncated GLI3 in control and Dyrk2 mutant cells.

DYRK2 was essential for the full reduction in GLI3 processing upon pathway stimulation with SAG (Figure 2G and $\mathrm{H}$ ). As the accumulation of GLI2 and GLI3 at ciliary tips is independent of DYRK2, and the regulation of GLI1 and GLI2 levels and GLI3 processing is dependent on DYRK2, we propose that DYRK2 is required for the $\mathrm{HH}$-regulated activation of GLI proteins at the ciliary tip.

\section{DYRK2 is required for skeletal development}

To explore the in vivo function of DYRK2, we generated a mutation in mouse Dyrk2 by CRISPR/Cas9-mediated deletion of the first two exons of Dyrk2, including the TATA box and initiator codon. (Figure 3A). In agreement with the results obtained using mutant NIH/3T3 cells, MEFs obtained from Dyrk2/- mice failed to induce Gli1 and Ptch1 in response to stimulation with SAG (Figure 3B and C).

No Dyrk2 ${ }^{--}$pups survived past two days of birth, indicating that DYRK2 is essential for perinatal viability. Truncation of the limbs was evident in Dyrk2 $2^{--}$pups at P0 (Figure 3D). The ratio of the length of $D y r k 2^{--}$limbs compared to the crown-to-rump measurement was significantly reduced as compared to control littermates (Figure 3E). Furthermore, rare instances of supernumerary digits were observed in Dyrk2 $2^{/-}$limbs (Supplemental figure 2A, 5/37 mutants assessed).

The reduction in limb elongation observed in Dyrk2 mutants is reminiscent of that caused by Ihh loss of function mutations (St-Jacques, Hammerschmidt et al. 1999). In Ihh mutant mice, defective chondrocyte differentiation causes defects in calcification of the long 
bones. We therefore assessed bone ossification of embryonic and neonatal Dyrk2 mutants utilizing alizarin red and alcian blue staining to measure calcified bone and cartilage, respectively (Figure 3F). There was a decrease in ossification in Dyrk2 ${ }^{-1}$ long bones at embryonic day (E) 16.5 relative to wild-type littermate controls (Figure 3G). Neonatal Dyrk2 mutants also exhibited reduced calcification of long bones (Supplemental figure 2B,C). Thus, DYRK2, like $\mathrm{IHH}$, is required for long bone growth and calcification.

\section{DYRK2 is a pharmacologically tractable candidate for the treatment of HH pathway- associated cancers}

Misactivation of $\mathrm{HH}$ signaling is critical for the formation of some cancers, including a subset of medulloblastomas (Goodrich, Milenković et al. 1997, Vorechovský, Tingby et al. 1997, Vŏrechovský, Undén et al. 1997, Zurawel, Allen et al. 2000, Taylor, Liu et al. 2002). To test whether DYRK2 might function in oncogenic $\mathrm{HH}$ signaling, we examined Dyrk2 expression in a mouse model of $\mathrm{HH}$-associated medulloblastoma caused by a constitutively active form of SMO (Xie, Murone et al. 1998, Mao, Ligon et al. 2006, Schüller, Heine et al. 2008). Dyrk2 expression and DYRK2 protein levels were increased in medulloblastomas (Figure 4A and B).

Pharmacological inhibition of the $\mathrm{HH}$ pathway is an effective therapy for some forms of HH pathway-associated cancers (Von Hoff, LoRusso et al. 2009). Therefore, we tested whether a pharmacological inhibitor of DYRK2 (Schmitt, Kail et al. 2014) could inhibit HH signaling in mouse embryonic fibroblasts (MEFs). DYRK2 inhibitor had no effect on SMO localization to cilia, but blocked SAG-dependent Gli1 and Ptch1 induction in a concentration dependent manner (Figure 4C). Pharmacological inhibition of other DYRK family members did not block $\mathrm{HH}$ signaling (Supplemental figure 3A). Moreover, DYRK2 inhibitor caused the MEFs to form longer cilia (Figure 4D). Thus, a small molecule inhibitor of DYRK2 reproduced the signaling and ciliary morphology effects of loss of function mutations in Dyrk2. These results reveal that evolutionary proteomics can help identify novel functional components of an organelle, in this case a ciliary-localized kinase critical for the activation of the $\mathrm{HH}$ signaling pathway. We anticipate that future analysis of ciliome members will uncover still other $\mathrm{HH}$ pathway regulators.

We predicted that proteins shared between the sea urchin and sea anemone ciliary proteomes, such as DYRK2, would function in ciliary biology. We further proposed that ciliary proteins conserved specifically among animals that employ $\mathrm{HH}$ signaling may function in $\mathrm{HH}$ signaling. In this work, we found that one such protein, DYRK2, localizes to cilia and determined, using a combination of CRISPR/Cas9-mediated knockout and pharmacological 
172 inhibition in both mammalian cells and mice, that DYRK2 positively regulates the HH pathway 173 downstream of SMO.

174 Little is known about how information is communicated between vertebrate SMO and the 175 GLI transcriptional effectors of the $\mathrm{HH}$ pathway. The identification of DYRK2 as epistatic to SMO 176 ciliary accumulation suggests that this kinase could function at this important and mysterious 177 step in $\mathrm{HH}$ signal transduction. Moreover, the requirement for DYRK2, not in ciliary tip 178 accumulation of GLI2 and GLI3, but in increasing GLI1 and GLI2 protein levels and in GLI3 processing, further points to DYRK2 as an important mediator of information between SMO and GLI proteins.

In addition to its requirement for $\mathrm{HH}$ signaling, DYRK2 limits the length of ciliary microtubules. Another member of the DYRK protein family, DYRK1A, phosphorylates $\beta$-Tubulin to regulate cytoplasmic microtubule dynamics (Ori-McKenney, McKenney et al. 2016). It will be interesting to test whether DYRK2 has an analogous role in the regulation of ciliary microtubules.

As DYRK2 is required for $\mathrm{HH}$ signal transduction in NIH/3T3 cells, it was surprising that Dyrk2 mutant embryos exhibit phenotypes that are reflective of moderate attenuation of $\mathrm{HH}$ signaling. For example, polydactyly is pronounced in embryos with strong defects in mouse embryos with strongly defective ciliary signaling, such as Tctn1 mutants (Garcia-Gonzalo, Corbit et al. 2011). Similarly, mutation of genes encoding some key components of the $\mathrm{HH}$ signal transduction pathway, such as Ptch1 or Kif7, cause completely penetrant polydactyly (Liem, He et al. 2009). In contrast, Dyrk2 mutants, displayed weakly penetrant polydactyly and bone growth and ossification defects. The Dyrk2 mutant phenotypes suggest tissue specific requirements or a modulatory role rather than a global requirement for $\mathrm{HH}$ signaling. It is possible that other dual-specificity kinases partially compensate for loss of DYRK2 in some cell types during development.

The defects in long bone growth and endochondral ossification of Dyrk2 mutants is reminiscent of those defects caused by loss of Ihh. Like Dyrk2 mutants, Ihh mutants display attenuated long bone growth and endochondral ossification (St-Jacques, Hammerschmidt et al. 1999). $\mathrm{IHH}$ is essential for the differentiation and proliferation of chondrocytes. The similarity of these phenotypes leads us to propose that DYRK2 transduces $\mathrm{IHH}$ signals in chondrocytes.

DYRK2 is overexpressed in a mouse model for medulloblastoma. The efficacy of in vitro 204 that pharmacologically inhibiting DYRK2 may be beneficial in the treatment of $\mathrm{HH}$ pathway205 associated cancers. As resistance to existing $\mathrm{HH}$ pathway inhibitors, such as vismodegib, can 
206

207

208

209

210

211

212

213

214

215

216

217

218

219

220

221

222

223

224

225

226

227

228

229

230

231

232

233

234

235

236

237

238

239

occur at the level of SMO (Yauch, Dijkgraaf et al. 2009, Brinkhuizen, Reinders et al. 2014, Pricl, Cortelazzi et al. 2015, Sharpe, Pau et al. 2015), inhibiting the pathway downstream of SMO at the level of DYRK2 may be beneficial in vismodegib-resistant cancers. Additionally, inhibition of DYRK2 may synergize with SMO inhibition. Thus, pharmacological inhibition of DYRK2 may be a viable strategy for treating $\mathrm{HH}$ pathway-associated cancers such as medulloblastoma and basal cell carcinoma.

\section{METHODS}

\section{CRISPR/Cas9-mediated mutagenesis}

CRISPR-mediated Dyrk2 mutations were generated as described previously (Ran, Hsu et al. 2013). Briefly, NIH/3T3-FIpln cells were transfected with pSpCas9(BB)-2A-Puro (PX458) containing one of two small guide sequences to target Dyrk2 (see Table S4 for primer sequences). 24 hours after transfection, selection media containing $300 \mathrm{ng} / \mathrm{ml}$ puromycin (InvivoGen) was applied to the cells for 48 hours. The cells were then cultured at low density and individual clones were isolated. To identify clones with mutations in Dyrk2, genomic DNA was extracted and primers specific to sequence flanking the small guide target sites were used to PCR amplify a 755bp portion of the Dyrk2 gene. The amplicons were sequenced by Sanger sequencing to identify mutations.

\section{Generation of Mouse Embryonic Fibroblast lines}

Isolation of MEF lines are adapted from (Jozefczuk, Drews et al. 2012). Briefly, pregnant mice at E15.5 were sacrificed, uterine horns were dissected and dipped in $70 \%$ ethanol and placed in ice-cold Delbecco's phosphate buffered saline. Embryos were extracted from the uterine horns, organs and heads were removed and the embryos were finely minced followed by digestion with Kunitz DNAse and .05\% Trypsin-EDTA. The resultant cell suspension was plated and grown to confluency in DMEM containing penicillin and streptomycin solution (Gibco). Cells were immortalized with SV40 virus and maintained.

\section{Alignments and dendrograms}

Alignment generated with ClustalW and Boxplot (Madeira, Park et al. 2019). Phylogenetic tree of the above sequences, calculated with NGPhylogeny.fr (Dereeper, Guignon et al. 2008, Lemoine, Correia et al. 2019). The NCBI accession numbers of the sequences were used in the alignments are as follows: NP_006473.2, NP_001014412.1, NP_001038298.1, XP_030856015.1, NP_001033810.1, XP_001638207.2,XP_002108931.1:14-400, 
XP_004990443.1, XP_015137468.1, XP_003224671.2, XP_030115200.1, and XP_026694893.1:34-604.

242

243 Mice

244 The Dyrk2 $2^{\text {em1(IMPC)lcs }}$ (Dyrk2) mouse allele was generated on the C57BL/6N background by the

245 International Mouse Phenotyping Consortium by CRISPR/Cas9-mediated deletion of the 246 proximal promoter and exons 1 and 2. B6.Cg-Tg(Atoh1-cre $)^{1 \text { BfrilJ }}$ (Math1-Cre), 247 GT(ROSA)26 $6^{\text {Sortm1(Smo/EYFP)Amc/J }}\left(S m o M 2^{\text {cond }}\right)$ and Ptch $1^{\text {tm1Mps/J }}\left(\right.$ Ptch $\left.1^{\circ}\right)$ alleles were obtained from 248 The Jackson Laboratory (Bar Harbor, ME). Math1-Cre SmoM2 ${ }^{\text {cond }}$ animals were monitored for 249 survival until death or protocol-defined neurologic endpoints including hydrocephalus or ataxia.

\section{Antibodies}

252 Primary antibodies used were anti- $\beta$-actin (60008-I-Ig, ProteinTech, Rosemont, IL and 907 ab8227, Abcam, Cambridge, MA), anti-acetylated a-tubulin (clone 6-11B-1, Sigma-Aldrich, St. Cell Signaling), anti-GLI2 for immunofluorescence (gift from J. Eggenshwiler (Cho, Ko et al. 2008)), anti-GLI2 for immunoblot (clone 1H6, gift from S. Scales (Wen, Lai et al. 2010)), antiGLI3 (clone 6F5, gift from S. Scales (Wen, Lai et al. 2010)), anti-SMO (ab38686, Abcam), and anti-ARL13B (clone N295B/66, Neuromab, Davis, CA and 17711-1-AP, ProteinTech), antiNinein (gift from J. Sillibourne, (Delgehyr, Sillibourne et al. 2005)), and anti- $\square$-tubulin (V-20, Santa Cruz Biotechnology).

\section{Ciliary length quantification}

265 To quantify cilia length in the Dyrk2 mutant and control cell lines, a freehand line was drawn

266 along the length of each cilium using FIJI software corresponding to the extent of ARL13B 267 immunofluorescent signal and the length of the line was measured. For WT and Mut1, data from 2683 experiments was compiled, 3 images per cell line. For Mut2, data from 2 experiments was 269 used (WT $n=82$, Mut1 $n=91$, Mut2 $n=41$ ). For control and DYRK2 inhibitor treated MEFs, 270 measurements were collected as described above from 1 experiment (untreated $n=125$, treated $271 \mathrm{n}=76$ ). The data was plotted using Prism 7 (Graph Pad). 
Mammalian cells were lysed using RIPA buffer (50mM Tris, $\mathrm{pH} 7.4,150 \mathrm{mM} \mathrm{NaCl}, 1 \% \mathrm{NP}-40$, $0.5 \%$ sodium deoxycholate) with protease inhibitors (Calbiochem, Billerica, MA). Protein samples were separated on 4-15\% gradient TGX precast gels (Bio-Rad, Hercules, CA). Protein was transferred to a nitrocellulose membrane (Whattman, Pittsburgh, PA). Membranes were blocked and antibodies were diluted in 5\% non-fat milk in TBST (50mM Tris, pH 7.6, 150mM $\mathrm{NaCl}, 0.1 \%$ Tween 20) and analyzed using ECL Lightening Plus (Perkin-Elmer, Waltham, MA).

\section{Quantitative RT-PCR}

RNA was extract from cells using the RNeasy Mini or Micro Kit (Qiagen, Hilden, Germany) and cDNA was synthesized using iScript cDNA Synthesis Kit (BioRad). Quantitative PCR was performed using EXPRESS SYBR GreenER with Premixed ROX (Invitrogen) and the 7900HT Fast Real-Time PCR System (ThermoFisher Scientific). Relative expression was calculated using the delta-delta CT method (Livak and Schmittgen 2001). For Dyrk2, Glyceraldehyde 3phosphate dehydrogenase (Gapdh) was used as the control for normalization. See Table S1 for primer sequences. The data were analyzed using Microsoft Excel and plotted using Prism7.

\section{Stimulation of cilia formation, Hh pathway activation and DYRK inhibition}

To induce cilia formation in confluent NIH/3T3 cells, cell culture medium was replaced with OptiMEM (CCF, UCSF) for at least 24 hours. To activate the Hh pathway, OptiMEM was supplemented with $1 \mu \mathrm{M}$ SAG (EMD-Millipore, Billerica, MA) for 24 hours.

To inhibit DYRK2, MEFs plated to confluency were treated in cell culture medium with the DYRK2 inhibitor compound 24 (Schmitt, Kail et al. 2014) (gift from M. Engel) for 24 hours. The culture medium was then replaced with OptiMEM containing compound 24 with or without $40 \mu \mathrm{M}$ SAG and incubated for 24 hours. DYRK1A was inhibited using $5 \mu \mathrm{M}$ harmine (R\&D Systems) and DYRK1B was inhibited using 5 $\mathrm{M}$ AZ191 (Tocris Bioscience, Bristol, United Kingdom) as described for compound 24.

\section{Bone preparations}

Embryonic and neonatal mice were euthanized, fixed overnight in 4\% PFA/PBS, and excess tissue was removed before dehydrating in ethanol. The alizarin red and alcian blue staining was performed as described in (Rigueur and Lyons 2014) followed by clearing in glycerol.

\section{Expression and in situ plasmid creation}


To construct the GFP-tagged expression plasmids for $S$. purpuratus DYRK2, gene specific cDNA was made from RNA of gastrula stage embryos using AccuScript (Agilent) or SuperScriptllI (Thermo Fisher Scientific), PCR amplified using primers containing the Spel and AsiSI cut sites and TOPO-TA cloned into PCR2.1 (Invitrogen) and inserted into the pCS2+8CeGFP (Gökirmak et al., 2012).

M. musculus Dyrk2 cDNA was cloned into pcDNA4/TO-C-3xFLAG using the In-fusion HD cloning system (Clontech). Dyrk2 cDNA clone \#6808145 was purchased (ThermoFisher Scientific). Site directed mutagenesis was performed using the QuikChange Lightning Kit (Agilent) to generate the DYRK2 kinase dead constructs. All primer sequences are listed in Table S1.

\section{FIGURE LEGENDS}

\section{Figure 1: DYRK2 is a conserved ciliary kinase that controls ciliary length}

A) Phylogeny of DYRK2 and $\mathrm{HH}$ and $\mathrm{SMO}$, key components of the $\mathrm{HH}$ signaling pathway. B) Immunofluoresence imaging of RPE-1 cells expressing DYRK2-GFP. Images depict cells stained for DYRK2-GFP (GFP, green) and cilia (ARL13B, red). C) Immunofluorescence imaging of IMCD3 cells expressing mammalian wild type DYR2K-FLAG or versions of DYRK2-FLAG incorporating kinase inactivating mutations K249R or K348M. Images depict cells stained for DYR2K-FLAG (FLAG, green), cilia (ARL13B, red), and nuclei (Hoechst, blue). Scale bar=2.5 $\mu \mathrm{m}$ D) Immunoblot for DYRK2 protein in wild type NIH/3T3 cells and frameshift mutant lines (Mut1 and Mut2). $\beta$-actin immunoblot serves as a loading control. E) Immunofluoresence imaging of serum-starved NIH/3T3 cells stained for the ciliary membrane (ARL13B, red), ciliary axoneme (acetylated tubulin [TUB ${ }^{A C}$, green) and nuclei (Hoechst, blue). Scale bar: $5 \mu \mathrm{m}$ F) Quantification of ciliary length in wild type, Dyrk2-mutant Mut1 and Mut2 NIH/3T3 cells. Dotted lines correspond to the mean and quartiles. A p value less than 0.05 was considered statistically significant and is denoted as follows: ${ }^{* * *} p<0.0001,{ }^{* * *} p<0.001,{ }^{* *} p<0.01,{ }^{*} p<0.05$, ns $=$ not significant. Significance was assessed using ordinary one-way ANOVA.

\section{Figure 2: DYRK2 is required for $\mathrm{HH}$ signal transduction}

A,B) qRT-PCR quantification of levels of $\mathrm{HH}$ targets Gli1 (A) and Ptch1 (B) in serum-starved $\mathrm{NIH} / 3 \mathrm{~T} 3$ cells stimulated with SAG or vehicle control (DMSO). Box and whisker plots indicate the minimum and maximum values, second and third quartiles, and median values. C-E) Immunofluorescent images of wild type and mutant NIH/3T3 cell lines. (C) Trafficking of SMO (green) to the cilia, marked with ARL13B (red), under SAG stimulation. (D and E) Trafficking of 
341 GLI2 (D) and GLI3 (E) (green) to the ciliary tips in response to SAG stimulation. Basal bodies

342 (gray) are labeled with Ninein (C) or $\square$-tubulin (D), cilia labeled with ARL13B (red), nuclei

343 labeled with Hoechst (blue). Scale bar: $5 \mu$ m. F,G) Immunoblots for GLI1, GLI2, and GLI3 in wild

344 type and Dyrk2-mutant Mut1 and Mut2 cell lines. $\beta$-actin immunoblot serves as a loading

345 control. (G) Immunoblot showing full-length GLI3 (FL) and the processed repressor form of GLI3

$346(\mathrm{R})$ stimulated with SAG or vehicle control (DMSO). H) Quantification of ratio of full-length to

347 repressor forms of GLI3 cells with and without SAG stimulation.

\section{Figure 3: DYRK2 is essential for mouse limb development}

350 A) Diagram of the CRISPR/Cas9-mediated excision of the first two exons of Dyrk2 in mouse.

351 The bent arrow indicates the direction of transcription from the transcriptional start site in exon 1

352 while the small arrowheads represent Cas9/sgRNA target sites. Pink boxes represent coding

353 regions of the Dyrk2 gene and grey boxes indicate the 5' and 3' UTRs. The dashed lines

354 indicate the region deleted via CRISPR/Cas9 mutagenesis. B,C) Box and whisker plots of qRT-

355 PCR of Dyrk2 ${ }^{-/}$and littermate control MEF lines treated with SAG or vehicle control (DMSO) for

$356 \mathrm{HH}$ target genes Gli1 (B) and Ptch1 (C). D) Gross images of neonatal (P0) Dyrk2 ${ }^{/-}$and

357 littermate control mice. Scale bar: $5 \mathrm{~mm}$. E) Quantification of the ratio of the length of the limb

358 (stylopod plus zeugopod) to the crown-rump length of Dyrk2 $2^{/-}$and littermate control mice.

359 Significance was assessed using two-tailed t-test. F) Wholemount images of alizarin red and

360 alcian blue-stained bone preparations of limbs from E16.5 Dyrk2 ${ }^{/-}$and Dyrk2 ${ }^{-/+}$littermate control

361 mice. G) Quantitation of endochondral ossification, measured by the ratio of the extent of

362 alizarin red staining to the length of the bone. Significance was calculated using multiple

363 unpaired t-tests with the Holm-Šídák test.

364

365

Figure 4: DYRK2 is a potentially druggable target in $\mathrm{HH}$ pathway-associated cancers

366

A) PCR measurement of Dyrk2 expression in control $\left(S m o M 2^{c o n d} / w t\right)$ mouse cerebellum tissue and medulloblastoma tumor (Math1-Cre SmoM2cond/wt) tissue. Error bars represent SEM, 4 mice per condition. B) Immunoblot for DYRK2 of control cerebellar and medulloblastoma tumor lysates. $\beta$-actin immunoblot serves as a loading control. C,D) qRT-PCR measurement of Gli1

370 and Ptch1 expression in MEFs in the presence of the indicated concentrations of compound 24

371 (Schmitt et al., 2014), a DYRK2 inhibitor, without stimulation (DMSO) or after stimulation with

372 SAG. The DYRK2 inhibitor blocks HH pathway activity in a concentration dependent manner.

373 The experiment was repeated 3 times and the data shown are representative from 1 
374 experiment. Error bars show SD of 2 technical replicates. E) Quantification of ciliary length in

$375 \mathrm{NIH} / 3 \mathrm{~T} 3$ cells treated with DYRK2 inhibitor.

Figure S1: Evolutionary conservation of Dyrk2

A) Alignment of protein sequences of human (H. sapiens), mouse (M. musculus), zebrafish ( $D$. rerio), sea urchin (S. pupuratus), sea squirt (C. intestinalis), sea anemone ( $N$. vectesis), placazoan ( $T$. adhaerens), fruit fly (D. melanogaster), chicken ( $G$. gallus), anole ( $A$. carolinensis), and zebrafinch (T. guttata), orthologs and closest homologous type II DYRK kinases. B) Dendrogram of the above sequences. Note that only partial $T$. adhaerens protein data is available and was excluded from dendrogram. C) Schematic of CRISPR mutations generated in NIH/3T3 cells. Mutations create frameshifts in the third exon.

Figure S2: DYRK2 inhibits digit formation and endochondral ossification

A) Wholemount images of forelimbs from a wild-type and mutant exhibiting preaxial polydactyly, stained with alizarin red and alcian blue. Scale bar: $1 \mathrm{~mm} \mathrm{B)}$ Wholemount images of alizarin red and alcian blue-stained bone preparations of limbs from P0 Dyrk2 $2^{/-}$and Dyrk2 ${ }^{/+}$littermate control mice. C) Quantitation of endochondral ossification, measured by the ratio of the extent of alizarin red staining to the length of the bone. Significance was calculated using multiple unpaired t-tests with the Holm-Šídák test.

Table S1: Oligonucleotides Used in Study

\begin{tabular}{|l|l|l|l|}
\hline Dyrk2 sg1 & $\begin{array}{l}\text { caccgCGACCAGGGATCGTAC } \\
\text { GTGC }\end{array}$ & $\begin{array}{l}\text { aaacGCACGTACGATCCCTGG } \\
\text { TCGC }\end{array}$ & $\begin{array}{l}\text { Used to generate NIH/3T3 } \\
\text { CRISPR mutants }\end{array}$ \\
\hline Dyrk2 sg2 & $\begin{array}{l}\text { caccgTGGTGGTTATGACGAC } \\
\text { GACC }\end{array}$ & $\begin{array}{l}\text { aacGGTCGTCGTCATAACCA } \\
\text { CCAC }\end{array}$ & $\begin{array}{l}\text { Used to generate NIH/3T3 } \\
\text { CRISPR mutants }\end{array}$ \\
\hline Dyrk2 sg3 & $\begin{array}{l}\text { caccgTCACGTGGCTTACAGG } \\
\text { TACG }\end{array}$ & $\begin{array}{l}\text { aaacCGTACCTGTAAGCCACG } \\
\text { TGAC }\end{array}$ & $\begin{array}{l}\text { Used to generate NIH/3T3 } \\
\text { CRISPR mutants }\end{array}$ \\
\hline Gli1 & $\begin{array}{l}\text { GGTGCTGCCTATAGCC } \\
\text { AGTGTCCTC }\end{array}$ & $\begin{array}{l}\text { GTGCCAATCCGGTGGA } \\
\text { GTCAGACCC }\end{array}$ & qPCR primer \\
\hline Ptch1 & $\begin{array}{l}\text { AATTCTCGACTCACTC } \\
\text { GTCCA }\end{array}$ & $\begin{array}{l}\text { CTCCTCATATTTGGGGC } \\
\text { CTT }\end{array}$ & qPCR primer \\
\hline Hprt & $\begin{array}{l}\text { CATAACCTGGTTCATCA } \\
\text { TCGC }\end{array}$ & $\begin{array}{l}\text { TCCTCCTCAGACCGCTT } \\
\text { TT }\end{array}$ & qPCR primer \\
\hline
\end{tabular}




\begin{tabular}{|l|l|l|lc|}
\hline $\begin{array}{l}\text { Dyrk2 WT } \\
\text { geno }\end{array}$ & $\begin{array}{l}\text { TCGAGGACAGGCCCCT } \\
\text { GCTA }\end{array}$ & Aggcagaccctggcttgttg & $\begin{array}{l}\text { Genotyping primer to } \\
\text { detect WT allele }\end{array}$ \\
\hline $\begin{array}{l}\text { Dyrk2 mut } \\
\text { geno }\end{array}$ & $\begin{array}{l}\text { TCGAGGACAGGCCCCT } \\
\text { GCTA }\end{array}$ & $\begin{array}{l}\text { TGCGTGGCTGAAATGA } \\
\text { AGGTGGA }\end{array}$ & $\begin{array}{l}\text { Genotyping primer to } \\
\text { detect mutant allele }\end{array}$ \\
\hline
\end{tabular}

400

401

402

403

404

405

406

407

408

409

410

411

412

413

414

415

416

417

418

419

420

421

422

423

424

425

426

427

428

429

430

431

432

433

434

435

436

437

438

439

440

441

\section{REFERENCES}

Aranda, S., A. Laguna and S. de la Luna (2011). "DYRK family of protein kinases: evolutionary relationships, biochemical properties, and functional roles." The FASEB Journal 25(2): 449-462. Brinkhuizen, T., M. G. Reinders, M. van Geel, A. J. Hendriksen, A. D. Paulussen, V. J. Winnepenninckx, K. B. Keymeulen, P. M. Soetekouw, M. A. van Steensel and K. Mosterd (2014). "Acquired resistance to the Hedgehog pathway inhibitor vismodegib due to smoothened mutations in treatment of locally advanced basal cell carcinoma." Journal of the American Academy of Dermatology 71(5): 1005-1008.

Caspary, T., C. E. Larkins and K. V. Anderson (2007). "The graded response to Sonic Hedgehog depends on cilia architecture." Developmental cell 12(5): 767-778.

Chen, J. K., J. Taipale, M. K. Cooper and P. A. Beachy (2002). "Inhibition of Hedgehog signaling by direct binding of cyclopamine to Smoothened." Genes \& development 16(21): 2743-2748.

Cho, A., H. W. Ko and J. T. Eggenschwiler (2008). "FKBP8 cell-autonomously controls neural tube patterning through a Gli2-and Kif3a-dependent mechanism." Developmental biology 321(1): 27-39.

Corbit, K. C., P. Aanstad, V. Singla, A. R. Norman, D. Y. Stainier and J. F. Reiter (2005). "Vertebrate Smoothened functions at the primary cilium." Nature 437(7061): 1018-1021.

Dai, P., H. Akimaru, Y. Tanaka, T. Maekawa, M. Nakafuku and S. Ishii (1999). "Sonic Hedgehog-induced Activation of the Gli1Promoter Is Mediated by GLI3." Journal of Biological Chemistry 274(12): 8143-8152.

Delgehyr, N., J. Sillibourne and M. Bornens (2005). "Microtubule nucleation and anchoring at the centrosome are independent processes linked by ninein function." Journal of cell science 118(8): 1565-1575.

Dereeper, A., V. Guignon, G. Blanc, S. Audic, S. Buffet, F. Chevenet, J.-F. Dufayard, S. Guindon, V. Lefort and M. Lescot (2008). "Phylogeny. fr: robust phylogenetic analysis for the non-specialist." Nucleic acids research 36(suppl_2): W465-W469.

Ericson, J., J. Briscoe, P. Rashbass, V. Van Heyningen and T. Jessell (1997). Graded sonic hedgehog signaling and the specification of cell fate in the ventral neural tube. Cold Spring Harbor symposia on quantitative biology, Cold Spring Harbor Laboratory Press.

Garcia-Gonzalo, F. R., K. C. Corbit, M. S. Sirerol-Piquer, G. Ramaswami, E. A. Otto, T. R. Noriega, A. D. Seol, J. F. Robinson, C. L. Bennett and D. J. Josifova (2011). "A transition zone complex regulates mammalian ciliogenesis and ciliary membrane composition." Nature genetics 43(8): 776.

Goodrich, L. V., L. Milenković, K. M. Higgins and M. P. Scott (1997). "Altered neural cell fates and medulloblastoma in mouse patched mutants." Science 277(5329): 1109-1113.

Haycraft, C. J., B. Banizs, Y. Aydin-Son, Q. Zhang, E. J. Michaud and B. K. Yoder (2005). "Gli2 and Gli3 localize to cilia and require the intraflagellar transport protein polaris for processing and function." PLoS genetics 1(4).

Hill, M. (1995). "Sonic hedgehog induces the differentiation of ventral forebrain neurons: a common signal for ventral patterning within the neural tube." Cell 81: 756. 
442

443

444

445

446

447

448

449

450

451

452

453

454

455

456

457

458

459

460

461

462

463

464

465

466

467

468

469

470

471

472

473

474

475

476

477

478

479

480

481

482

483

484

485

486

487

488

489

490

491

492

Jozefczuk, J., K. Drews and J. Adjaye (2012). "Preparation of mouse embryonic fibroblast cells suitable for culturing human embryonic and induced pluripotent stem cells." JoVE (Journal of Visualized Experiments)(64): e3854.

Kim, J., M. Kato and P. A. Beachy (2009). "Gli2 trafficking links Hedgehog-dependent activation of Smoothened in the primary cilium to transcriptional activation in the nucleus." Proceedings of the National Academy of Sciences 106(51): 21666-21671.

Lemoine, F., D. Correia, V. Lefort, O. Doppelt-Azeroual, F. Mareuil, S. Cohen-Boulakia and O. Gascuel (2019). "NGPhylogeny.fr: new generation phylogenetic services for non-specialists." Nucleic Acids Research 47(W1): W260-W265.

Liem, K. F., M. He, P. J. R. Ocbina and K. V. Anderson (2009). "Mouse Kif7/Costal2 is a ciliaassociated protein that regulates Sonic hedgehog signaling." Proceedings of the National Academy of Sciences 106(32): 13377-13382.

Livak, K. J. and T. D. Schmittgen (2001). "Analysis of relative gene expression data using realtime quantitative PCR and the 2- $\triangle \Delta C T$ method." methods 25(4): 402-408.

Maddika, S. and J. Chen (2009). "Protein kinase DYRK2 is a scaffold that facilitates assembly of an E3 ligase." Nat Cell Biol 11(4): 409-419.

Madeira, F., Y. m. Park, J. Lee, N. Buso, T. Gur, N. Madhusoodanan, P. Basutkar, A. R. N. Tivey, S. C. Potter, R. D. Finn and R. Lopez (2019). "The EMBL-EBI search and sequence analysis tools APIs in 2019." Nucleic Acids Research 47(W1): W636-W641.

Mao, J., K. L. Ligon, E. Y. Rakhlin, S. P. Thayer, R. T. Bronson, D. Rowitch and A. P. McMahon (2006). "A novel somatic mouse model to survey tumorigenic potential applied to the Hedgehog pathway." Cancer research 66(20): 10171-10178.

Matus, D. Q., C. R. Magie, K. Pang, M. Q. Martindale and G. H. Thomsen (2008). "The Hedgehog gene family of the cnidarian, Nematostella vectensis, and implications for understanding metazoan Hedgehog pathway evolution." Developmental biology 313(2): 501 518.

Ori-McKenney, K. M., R. J. McKenney, H. H. Huang, T. Li, S. Meltzer, L. Y. Jan, R. D. Vale, A. P. Wiita and Y. N. Jan (2016). "Phosphorylation of $\beta$-Tubulin by the down syndrome kinase, minibrain/DYRK1a, regulates microtubule dynamics and dendrite morphogenesis." Neuron 90(3): 551-563.

Pricl, S., B. Cortelazzi, V. Dal Col, D. Marson, E. Laurini, M. Fermeglia, L. Licitra, S. Pilotti, P. Bossi and F. Perrone (2015). "Smoothened (SMO) receptor mutations dictate resistance to vismodegib in basal cell carcinoma." Molecular oncology 9(2): 389-397.

Ran, F. A., P. D. Hsu, J. Wright, V. Agarwala, D. A. Scott and F. Zhang (2013). "Genome engineering using the CRISPR-Cas9 system." Nature Protocols 8(11): 2281-2308.

Rigueur, D. and K. M. Lyons (2014). Whole-mount skeletal staining. Skeletal Development and Repair, Springer: 113-121.

Rohatgi, R., L. Milenkovic and M. P. Scott (2007). "Patched1 regulates hedgehog signaling at the primary cilium." Science 317(5836): 372-376.

Sasaki, H., Y. Nishizaki, C.-c. Hui, M. Nakafuku and H. Kondoh (1999). "Regulation of Gli2 and Gli3 activities by an amino-terminal repression domain: implication of Gli2 and Gli3 as primary mediators of Shh signaling." Development 126(17): 3915-3924.

Schmitt, C., D. Kail, M. Mariano, M. Empting, N. Weber, T. Paul, R. W. Hartmann and M. Engel (2014). "Design and synthesis of a library of lead-like 2, 4-bisheterocyclic substituted thiophenes as selective Dyrk/Clk inhibitors." Plos one 9(3).

Schüller, U., V. M. Heine, J. Mao, A. T. Kho, A. K. Dillon, Y.-G. Han, E. Huillard, T. Sun, A. H. Ligon and Y. Qian (2008). "Acquisition of granule neuron precursor identity is a critical determinant of progenitor cell competence to form Shh-induced medulloblastoma." $\underline{\text { Cancer cell }}$ 14(2): 123-134.

Sharpe, H. J., G. Pau, G. J. Dijkgraaf, N. Basset-Seguin, Z. Modrusan, T. Januario, V. Tsui, A. B. Durham, A. A. Dlugosz, P. M. Haverty, R. Bourgon, J. Y. Tang, K. Y. Sarin, L. Dirix, D. C. 
524

525

526

527

528

529

530

531

532

533

534

535

536

537

538

Fisher, C. M. Rudin, H. Sofen, M. R. Migden, R. L. Yauch and F. J. de Sauvage (2015). "Genomic analysis of smoothened inhibitor resistance in basal cell carcinoma." Cancer cell 27(3): 327-341.

Sharpe, J., L. Lettice, J. Hecksher-Sørensen, M. Fox, R. Hill and R. Krumlauf (1999). "Identification of <em>Sonic hedgehog $</ \mathrm{em}>$ as a candidate gene responsible for the polydactylous mouse mutant <em $>$ Sasquatch</em>." Current Biology 9(2): S1-100.

Sigg, M. A., T. Menchen, J. Johnson, C. Lee, S. P. Choksi, G. Garcia, H. Busengdal, G. Dougherty, P. Pennekamp, C. Werner, F. Rentzsch, N. Krogan, J. B. Wallingford, H. Omran and J. F. Reiter (2017). "Evolutionary proteomics uncovers ciliary signaling components." bioRxiv: 153437.

St-Jacques, B., M. Hammerschmidt and A. P. McMahon (1999). "Indian hedgehog signaling regulates proliferation and differentiation of chondrocytes and is essential for bone formation." Genes \& development 13(16): 2072-2086.

Taylor, M. D., L. Liu, C. Raffel, C.-c. Hui, T. G. Mainprize, X. Zhang, R. Agatep, S. Chiappa, L. Gao and A. Lowrance (2002). "Mutations in SUFU predispose to medulloblastoma." Nature genetics 31(3): 306-310.

Varjosalo, M., M. Björklund, F. Cheng, H. Syvänen, T. Kivioja, S. Kilpinen, Z. Sun, O. Kallioniemi, H. G. Stunnenberg and W.-W. He (2008). "Application of active and kinase-deficient kinome collection for identification of kinases regulating hedgehog signaling." Cell 133(3): 537548.

Von Hoff, D. D., P. M. LoRusso, C. M. Rudin, J. C. Reddy, R. L. Yauch, R. Tibes, G. J. Weiss, M. J. Borad, C. L. Hann and J. R. Brahmer (2009). "Inhibition of the hedgehog pathway in advanced basal-cell carcinoma." New England Journal of Medicine 361(12): 1164-1172.

Vorechovský, I., O. Tingby, M. Hartman, B. Strömberg, M. Nister, V. Collins and R. Toftgård (1997). "Somatic mutations in the human homologue of Drosophila patched in primitive neuroectodermal tumours." Oncogene 15(3): 361-366.

Vŏrechovský, I., A. B. Undén, B. Sandstedt, R. Toftgård and M. Ståhle-Bäckdahl (1997). "Trichoepitheliomas contain somatic mutations in the overexpressed PTCH gene: support for a gatekeeper mechanism in skin tumorigenesis." Cancer research 57(21): 4677-4681.

Wang, B., J. F. Fallon and P. A. Beachy (2000). "Hedgehog-regulated processing of Gli3 produces an anterior/posterior repressor gradient in the developing vertebrate limb." $\underline{\text { Cell 100(4): }}$ 423-434.

Warner, J. F., A. M. McCarthy, R. L. Morris and D. R. McClay (2014). "Hedgehog signaling requires motile cilia in the sea urchin." Molecular biology and evolution 31(1): 18-22.

Wen, X., C. K. Lai, M. Evangelista, J.-A. Hongo, F. J. de Sauvage and S. J. Scales (2010). "Kinetics of hedgehog-dependent full-length Gli3 accumulation in primary cilia and subsequent degradation." Molecular and cellular biology 30(8): 1910-1922.

Xie, J., M. Murone, S.-M. Luoh, A. Ryan, Q. Gu, C. Zhang, J. M. Bonifas, C.-W. Lam, M. Hynes and A. Goddard (1998). "Activating Smoothened mutations in sporadic basal-cell carcinoma." Nature 391(6662): 90-92.

Yauch, R. L., G. J. Dijkgraaf, B. Alicke, T. Januario, C. P. Ahn, T. Holcomb, K. Pujara, J. Stinson, C. A. Callahan and T. Tang (2009). "Smoothened mutation confers resistance to a Hedgehog pathway inhibitor in medulloblastoma." Science 326(5952): 572-574.

Zurawel, R. H., C. Allen, R. Wechsler-Reya, M. P. Scott and C. Raffel (2000). "Evidence that haploinsufficiency of Ptch leads to medulloblastoma in mice." Genes, Chromosomes and Cancer 28(1): 77-81. 
bioRxiv preppint dqi: https://doi.org/10.1101/2020.08.31.275511; this version posted August 31, 2020. The copyright holder for this preprint

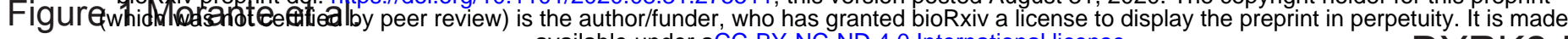
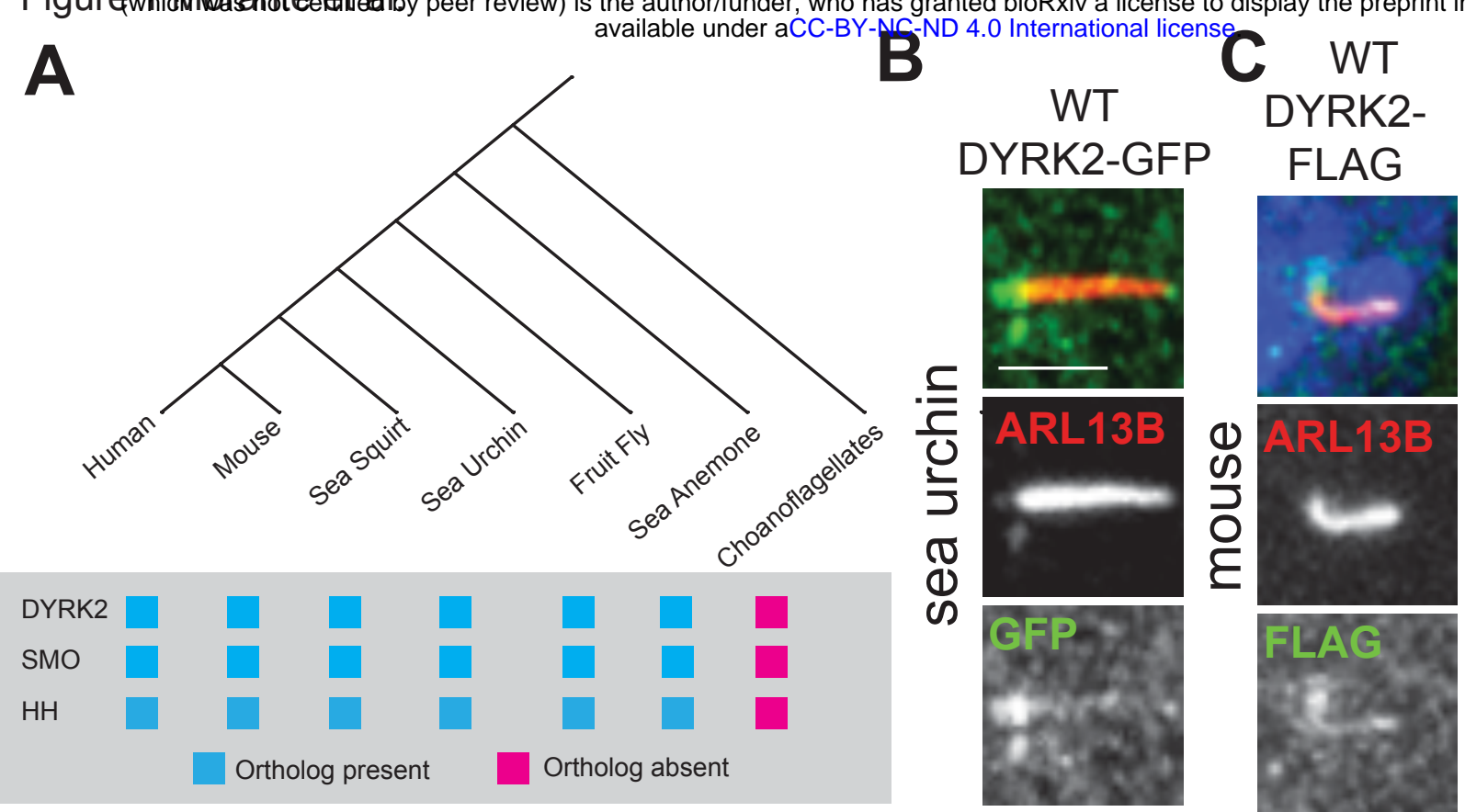

DYRK2-FLAG kinase dead

D

Dyrk2 $2^{-/-}$

WT Mut1 Mut2

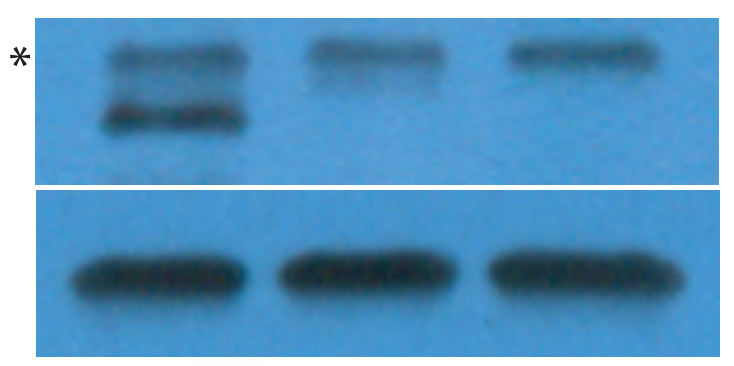

\section{DYRK2}

$\beta$-actin

$F$

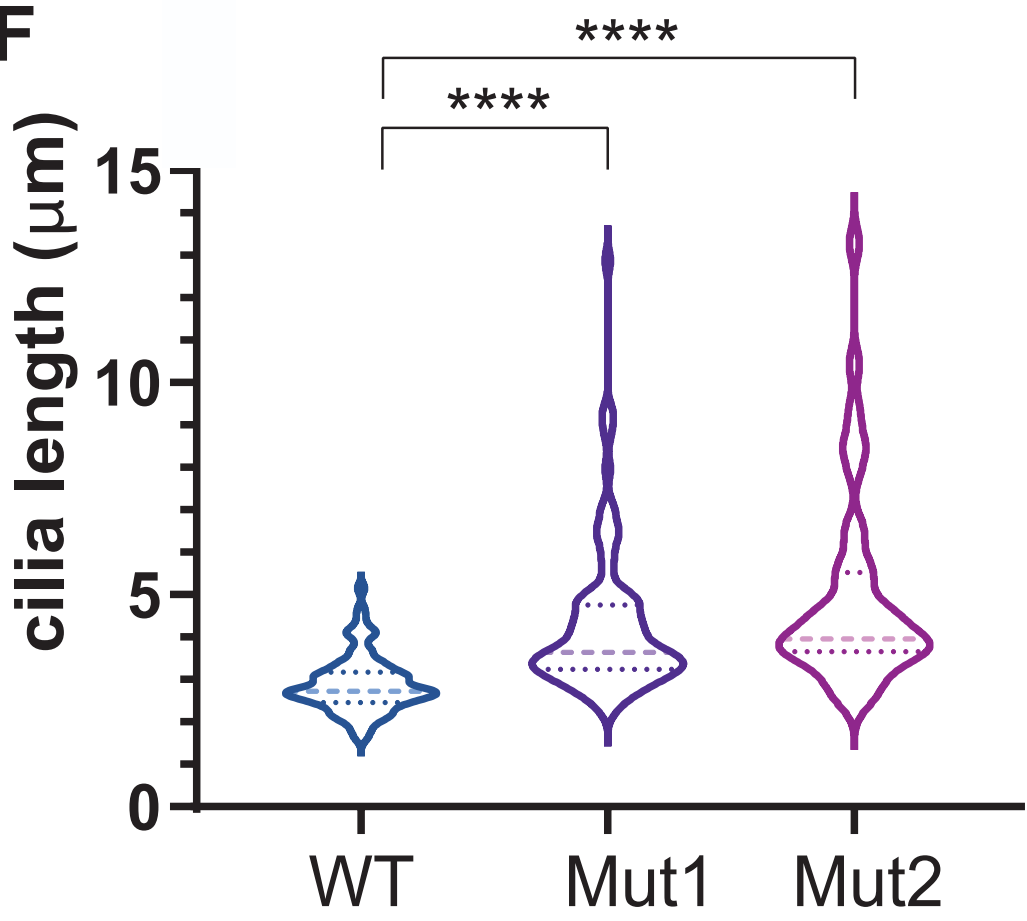

Dyrk2 $2^{-/-}$

E
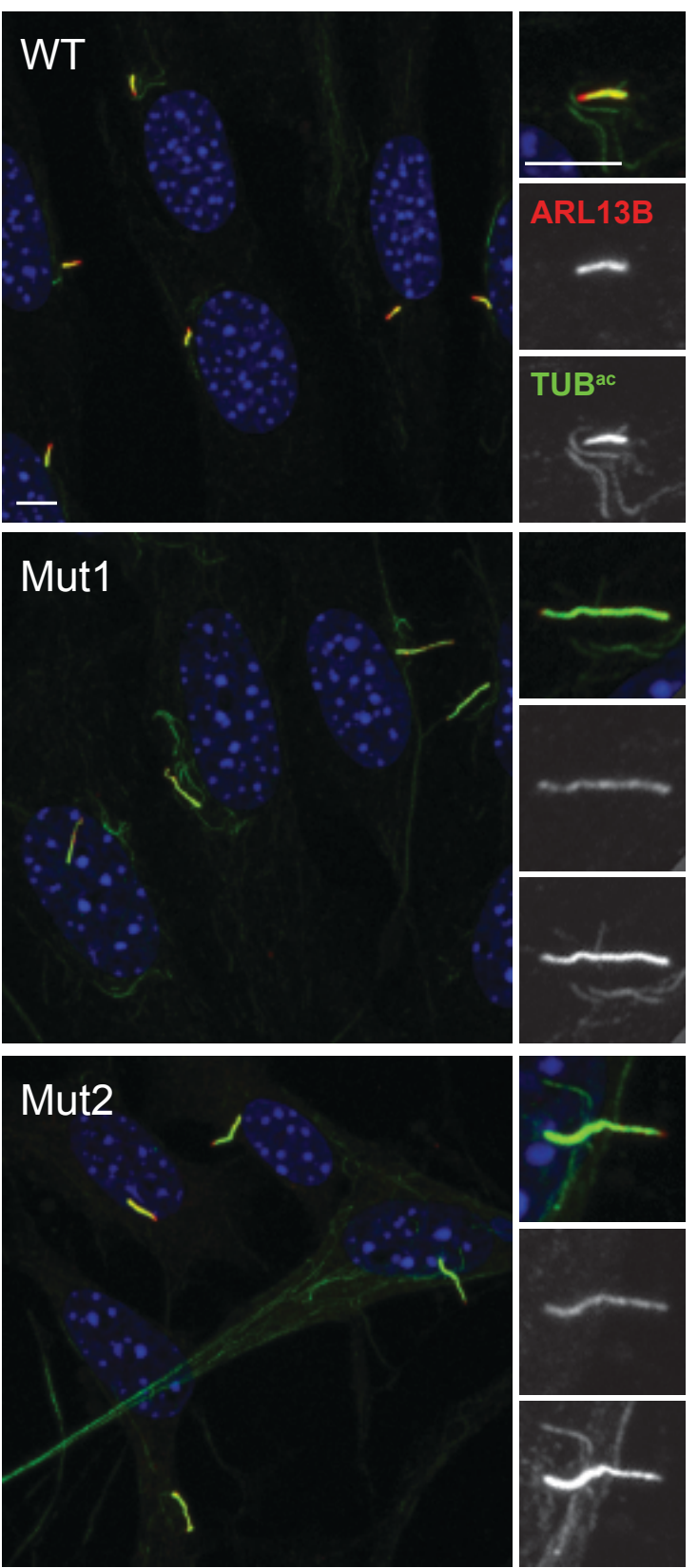


\section{A}

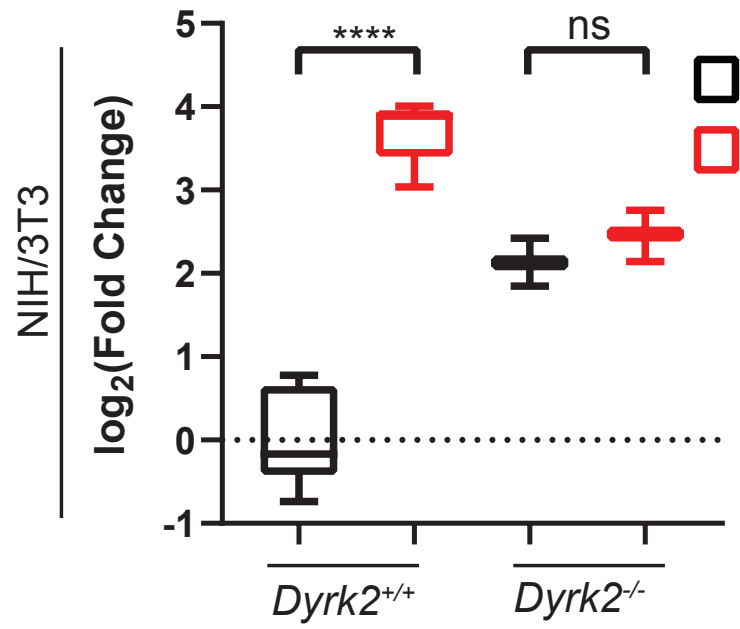

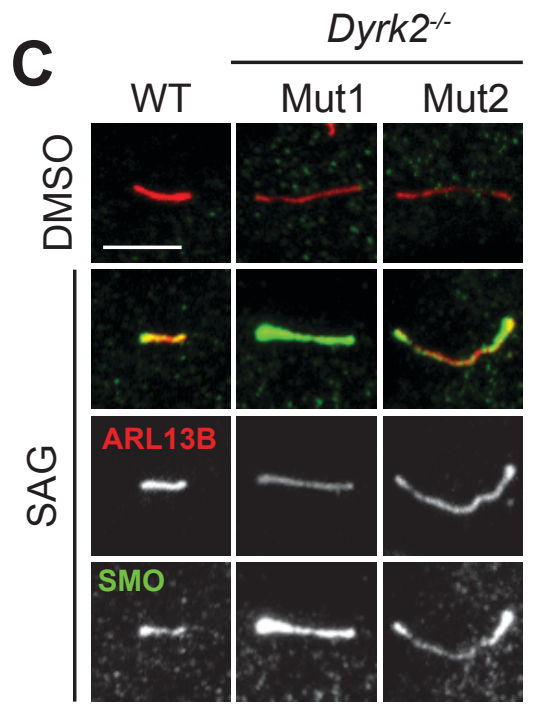

$\mathbf{F}$

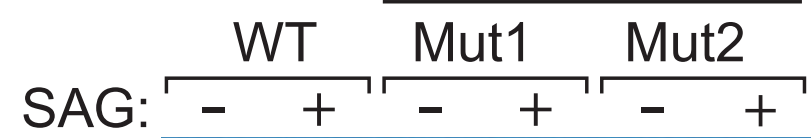
GLI1 $\beta$-actin GLI2-FL $\beta$-actin
Ptch1 mRNA

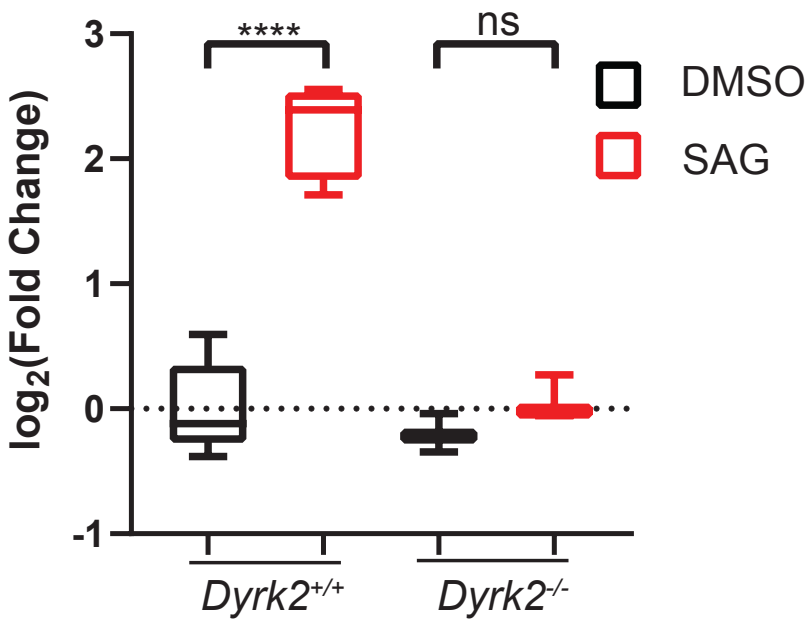

DMSO SAG

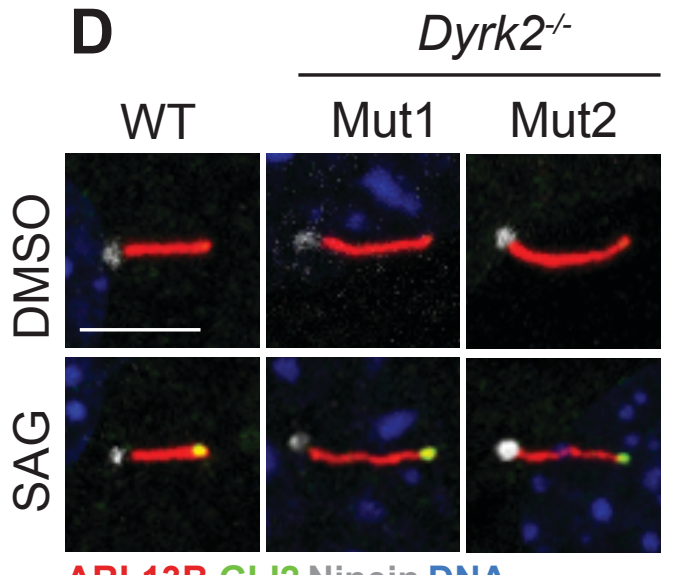

E

Dyrk2--

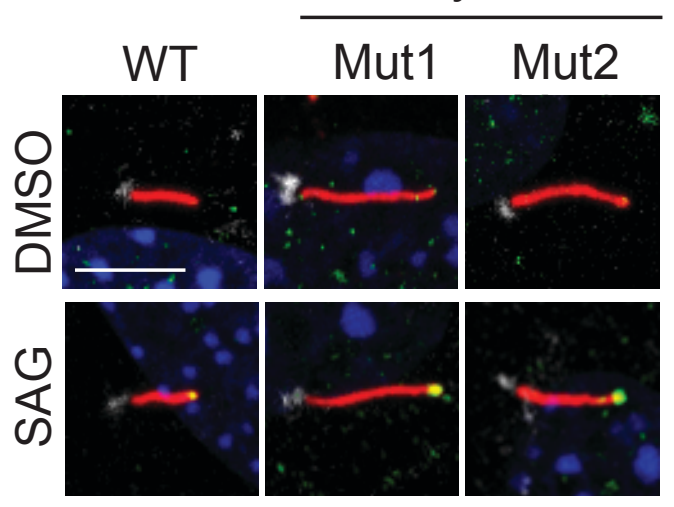

ARL13B GLI3 YTUB DNA

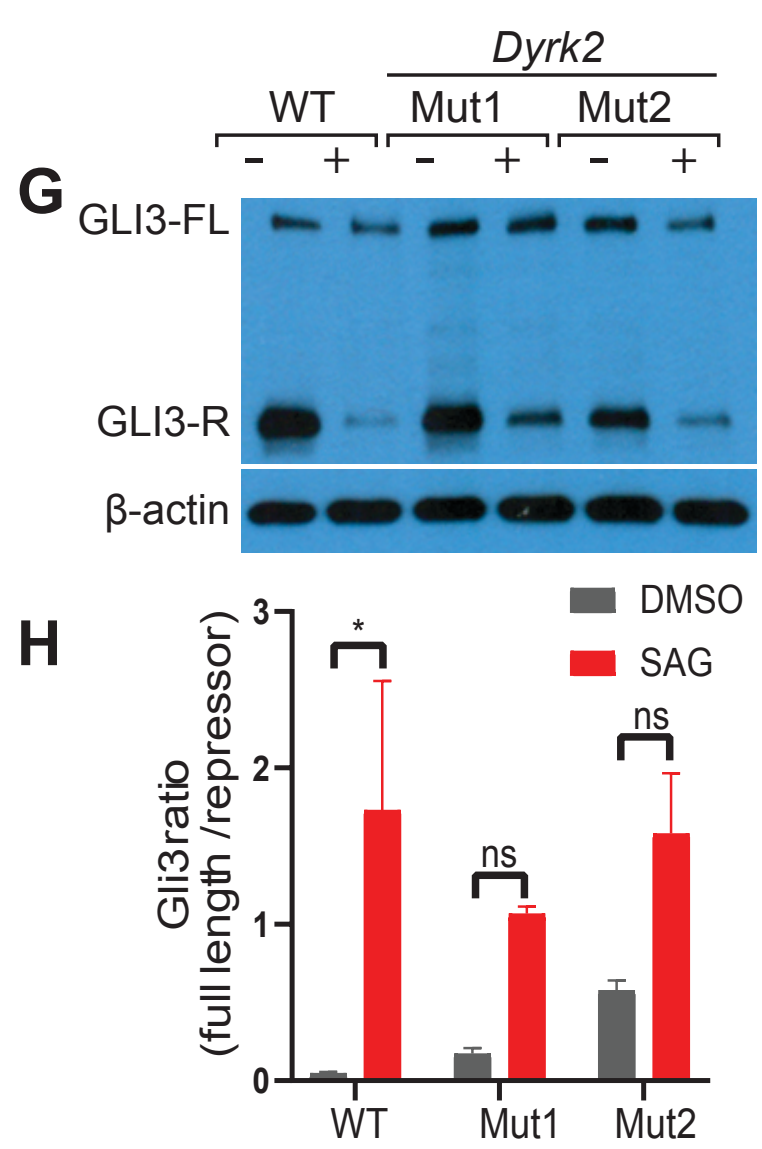


bioBxis preprint,doi: https://doi.org/10.1101/2020.08.31.275511; this version posted August 31, 2020. The copyright holder for this preprint

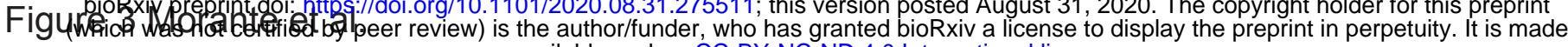

A available under aCG-BY-NC-ND 4.0 International license.

Wild-type

Dyrk2

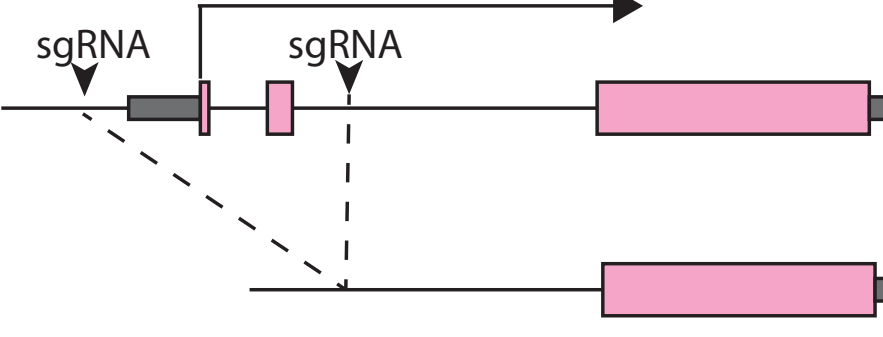

B

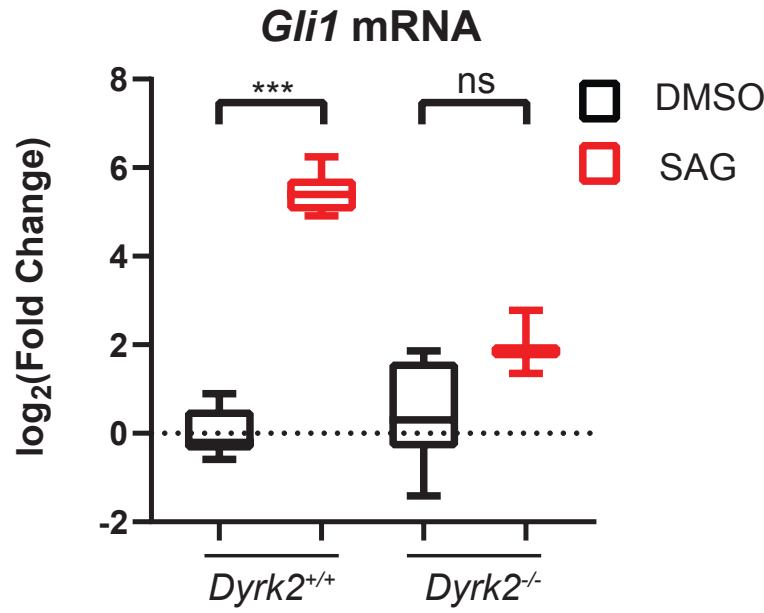

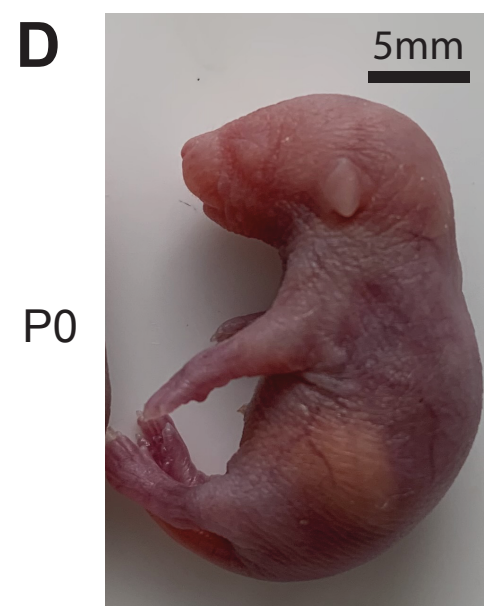

Dyrk2+/+

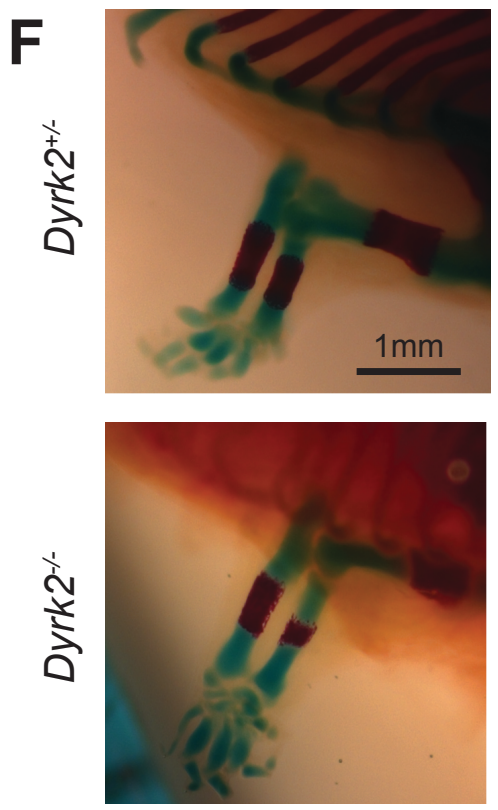

Forelimb

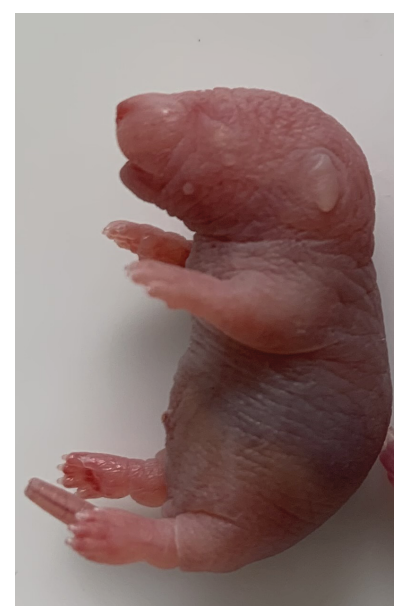

Dyrk2-1-
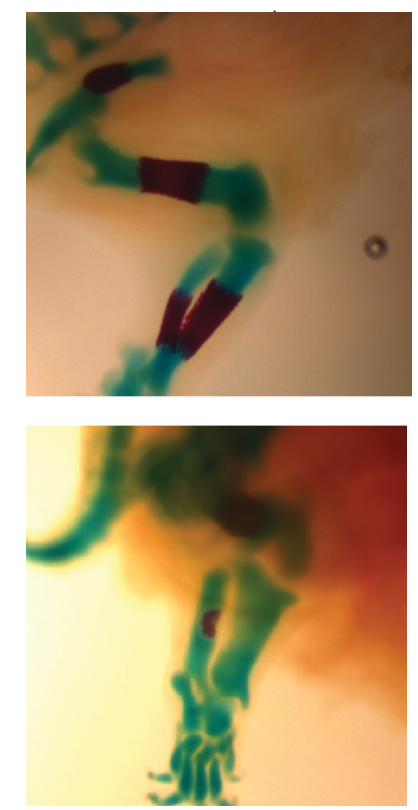

Hindlimb
G

E

Dyrk2 $^{+/+}$Dyrk2 ${ }^{-/-}$
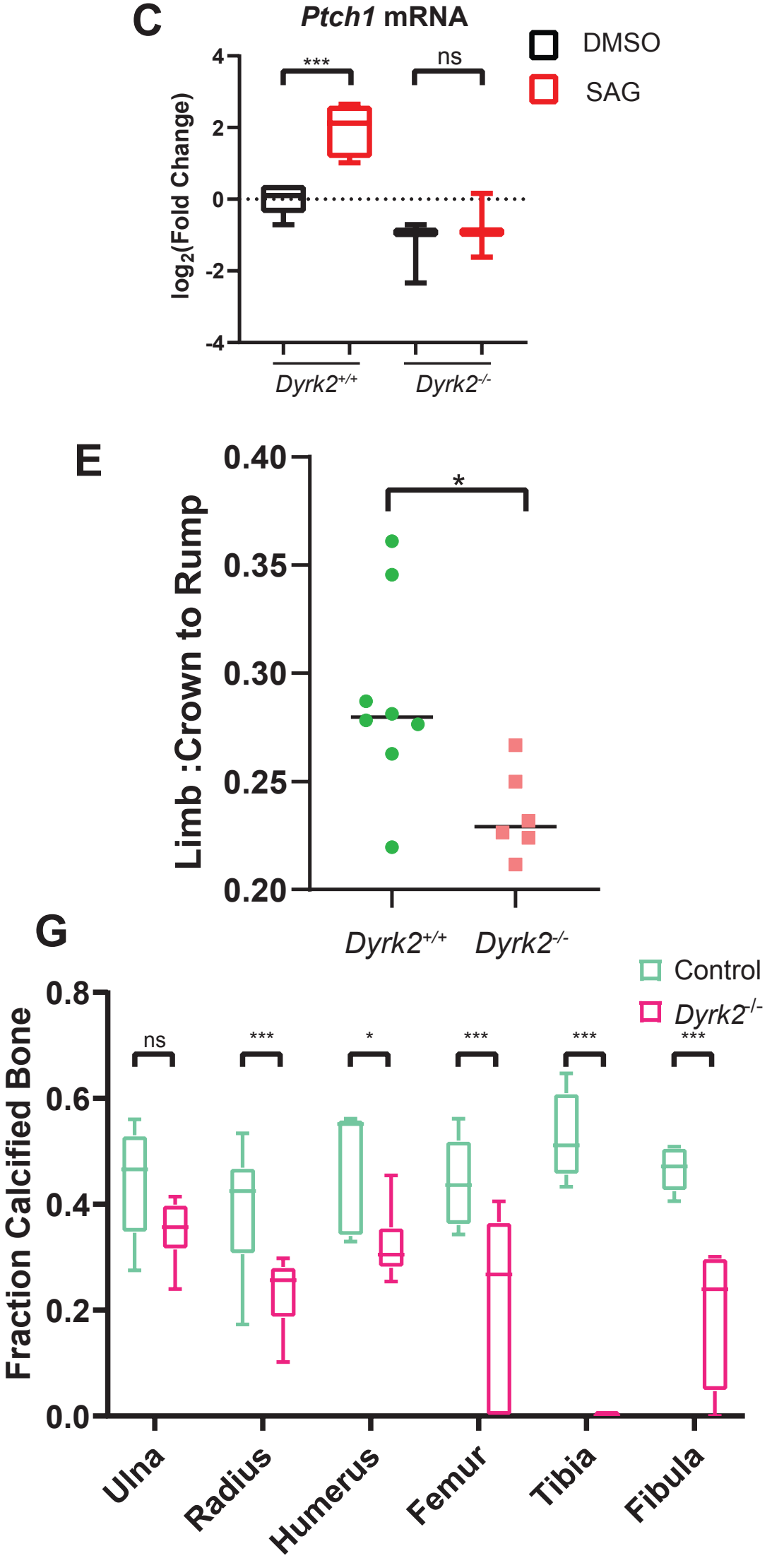
A
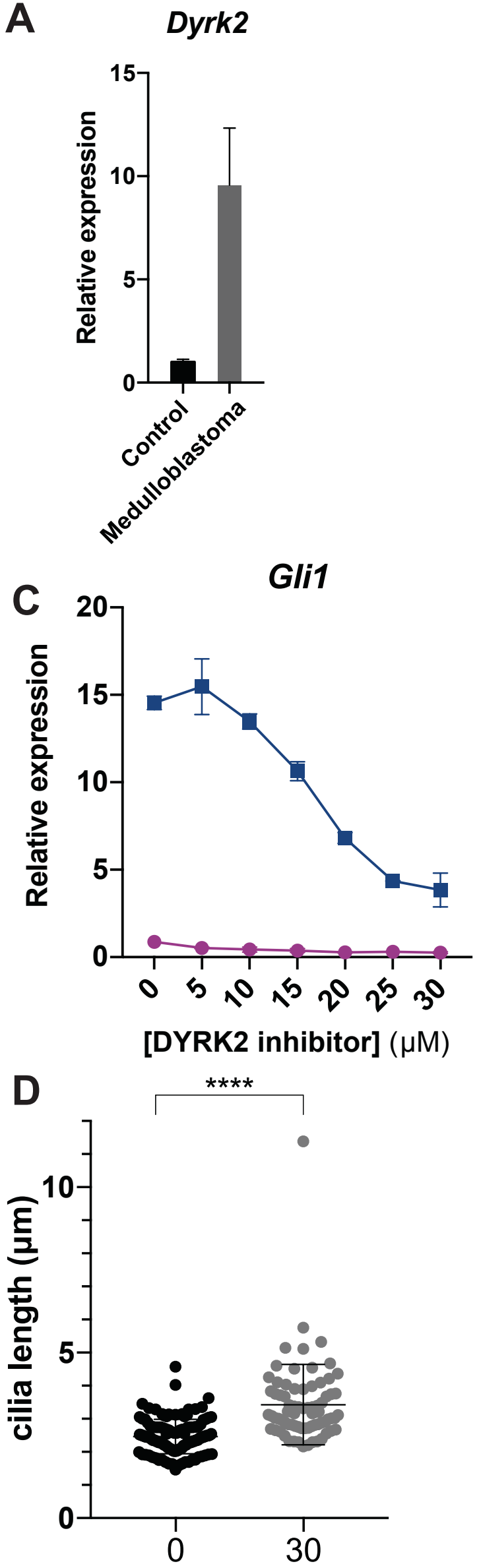

[DYRK2 inhibitor] ( $\mu \mathrm{M})$
B
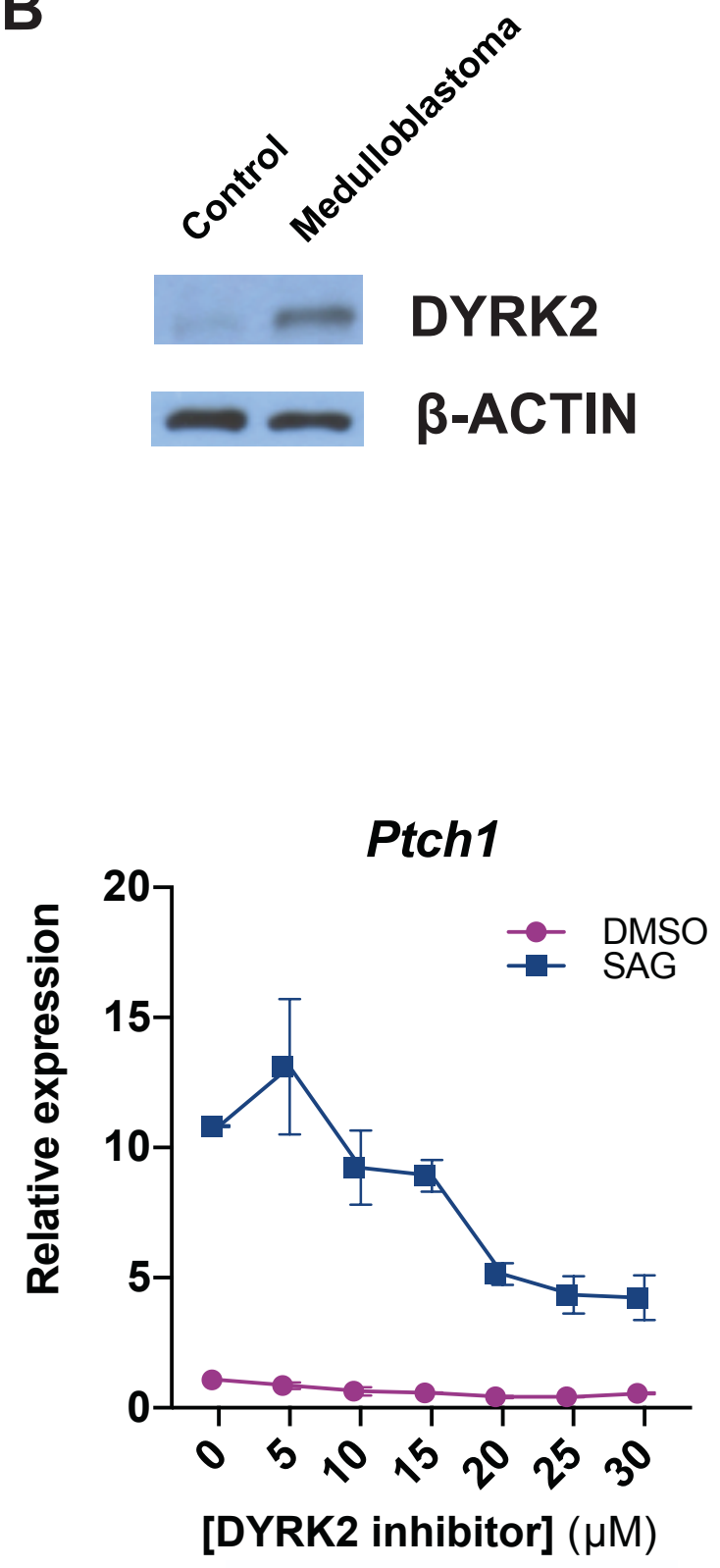